\title{
Las nuevas tecnologías como mediadoras en el desarrollo de la competencia científica
}

The new technologies as mediators in the development of the scientific skills

Dor Marina Daza P., Nohora Carolina Lozano R. y Martha Villarreal

Universidad Pedagógica Nacional de Colombia. maridape26@hotmail.com, natube14@yahoo.es, millarreal@pedagogica.edu.co

\section{Resumen}

La didáctica de la Química es un área que ha evolucionado con la introducción de las nuevas tecnologías en todos los campos del saber. Es por esto que el objetivo principal de este trabajo se centra en el desarrollo de competencias científicas mediante el uso de las nuevas tecnologías (calculadoras graficadoras voyage, 200) en el proceso pedagógico escolar. Esto hace necesario diseñar e implementar una serie de actividades que permitan realizar el trabajo práctico desde una perspectiva de la resolución de problemas de situaciones relacionadas con la vida cotidiana y conceptos químicos que además propicien el desarrollo de competencias científicas en la interpretación de situaciones y en el establecer condiciones, para la resolución de problemas.

\section{Palabras claves}

Competencia Científica, Nuevas Tecnologías (calauladoras graficadoras), resolución de problemas

\section{Abstract}

The teaching of chemistry is an area that has evolved with the establishment of new technologies in all knowledge fields. For this reason the main objective of this work is focused in the development of scientific skills goals through the use of new technology (graphing calculator voyage 200 ) in the educational process at school. This makes it necessary to design and apply practical guide that allows the student confront problems related with different situations, in everyday life and chemical concepts, besides, it support the development of scientific competence in the interpretation of situations and to establish conditions for the resolution of problems.

\section{Keywords}

Science Achievement, New Technologies (graphing calaulators), solving problems

\section{Introducción}

Las nuevas tecnologías de la información y la comunicación (NTIC) lo han cambiado todo, la forma de vivir, de trabajar, de producir, de comunicamos, de comprar, de vender, de enseñar, de aprender. Todo el entomo es distinto. El gran imperativo es preparamos y aprender a vivir en ese nuevo entomo.

Hoy, dice Cardona, (2002), educar es gobemar, así era en el siglo XIX, así fue durante el siglo XX y así será a medida que avance el nuevo siglo. Este va a ser el siglo del saber, el siglo de la 
racionalidad científica y tecnológica. Cierto, nuestra especie ha dependido siempre de sus creencias y sus tecnologías, pero ahora esas creencias van siendo cada vez mas penetradas por la ciencia y esas tecnologías están cambiando a un ritmo sin precedentes.

La tecnología está influenciando al menos en dos aspectos al mundo educacional: Uno relacionado con los intereses pedagógicos, administrativos y de gestión escolar y el segundo con los cambios en las habilidades y competencias requeridas, para lograr una inserción de las personas en la sociedad actual. (Villarreal, 2003).

Actualmente la influencia que tiene la tecnología en la Educación como medio facilitador tanto en el proceso de aprendizaje como en el de enseñanza de algunas temáticas de las ciencias, ha generado su indusión en las instituciones educativas como es el caso, de las calculadoras graficadoras (voyage 200). No obstante, en general son varios los profesores que aunque cuentan con este tipo de recursos para desarrollar sus dases, se abstienen de utilizarlos porque no saben cómo ni en qué momento hacerlo. Por tanto, se presentan en este proyecto, algunas estrategias para abordar ciertas temáticas de la química escolar, en donde sea factible el uso de herramientas básicas de la calculadora, como mediadoras en el desarrollo de competencias cient́ficas como interpretar situaciones y establecer condiciones.

El tema de las competencias científicas que se pretende desarrollar en nuestro proyecto, tiene dos horizontes de análisis: el que se refiere a las competencias científicas requeridas para hacer ciencia y el que se refiere a las competencias científicas que sería deseable desarrollar en todos los ciudadanos, independientemente de la tarea social que desempeñarán. Sin duda las competencias que caracterizan a unos y a otros no son exduyentes y tienen muchos elementos comunes, pero el segundo tipo de competencias interesa especialmente a la educación básica y media porque tiene relación con la vida de todos los ciudadanos (Hemández, 2005). Para lograr esos objetivos las propuestas educativas deben permitirles a los estudiantes apropiarse de capacidades (saberes, habilidades, valores, actitudes y comportamientos) necesarias para enfrentar exitosamente contextos y problemas de la vida cotidiana, privada y social.

Y con la esperanza de aportar en este proceso, queremos mejorar la calidad de la educación básica en nuestras instituciones, y propiciar la búsqueda de nuevos procesos de enseñanza de las ciencias y la tecnología, así como de procesos que favorezcan la formación de una sociedad en mejores condiciones para abordar la competitividad internacional, con una calidad de vida más digna, se pretende utilizar artefactos tecnológicos que ayuden a orientar el proceso de enseñanza de la química en el aula de dase y que a la vez favorezca el desarrollo de competencias científicas en los estudiantes.

Reconocemos que una ventaja directa de la tecnología en nuestro campo educativo, es la posibilidad que ofrecen para la simulación de fenómenos, sobre los auales los alumnos puedan trabajar sin riesgo de ningún tipo, observar los elementos significativos de una actividad, proceso 0 fenómeno, o descomponer un producto en sus partes o en el proceso seguido para su elaboración y de esta manera va reconstruyendo su conocimiento y a la vez desarrolla habilidades para saber para llegar a la solución de alguna problemática planteada.

\section{Desarrollo}

Problema: Es de conocimiento general que la enseñanza de las ciencias enfatiza la calidad de los procesos, y debe estar dirigida, no tanto a la exactitud con que se manejen los conceptos espeć́icos, sino a las actitudes críticas con las que los estudiantes aprenden a juzgar estos conceptos, por lo tanto al realizar un análisis de los resultados obtenidos en las pruebas en que se evalúan las competencias en química, encontramos que en nuestros estudiantes se evidencia 
falencias en competencias científicas como la interpretación de situaciones y el establecer condiciones, para la resolución de problemas. Es por ello que nuestro proyecto plantea la siguiente problemática

Qué implicaciones tendrá la implementación de una estrategia pedagógica y didáctica, que induya el uso de calculadoras (voyage 200), en el desarrollo de competencia científica en los colegios distritales "Gustavo Rojas Pinilla y Virginia Gutiérrez de Pineda"

Objetivo. El desarrollo de competencias científicas, mediante la implementación de una estrategia pedagógica y didáctica en la aual se haga el uso de las nuevas tecnologías (calculadoras graficadoras voyage 200 ) en el proceso pedagógico escolar.

Hipótesis de trabajo. La aplicación a los estudiantes de los colegios distritales General Gustavo Rojas Pinilla y Virginia Gutiérrez de Pineda de esta estrategia pedagógica y didáctica, que incluya el uso de calculadoras (voyage 200), facilitará el desarrollo de competencias en la interpretación de situaciones y en el establecer condiciones, para la resolución de problemas.

Metodología. La investigación es de tipo ausi-experiemntal, sin grupo control. Se llevará a cabo en tres fases, a saber: La fase de diagnóstico en la que se aplicaran algunos instrumentos para determinar el estado de las competencias antes de la aplicación de la estrategia. La segunda fase consisten el diseño e implementación de una estrategia basada en la resolución de problemas y una tercera fase de evaluación.

Primera fase: diagnostico

En esta etapa se realiza el diseño de algunos instrumentos (pretest), que permitan recolectar información sobre las ideas previas de los estudiantes acerca del nivel conceptual (gases, pH, manejo de calculadoras voyage 200) y del nivel de sus competencias científicas espeáficas como interpretar situaciones, establecer condiciones y formular hipótesis y regularidades. De esta manera se determinara una nivelación de los conceptos si se requiere. Se espera que los resultados obtenidos evidencien bajo desarrollo en estas competencias.

\section{Segunda fase: diseño e implementación}

En esta fase se diseñaran actividades en las aules algunas experiencias montadas para el uso de calculadoras Voyage $\mathbf{2 0 0}$ de forma tradicional, serán traducidas a situaciones problema desde las cuales los estudiantes deberán partir realizando entre otras cosas la delimitación de problemas, la revisión conceptual, la formulación de hipótesis, la generación de diseños experimentales que induyan el uso de las calculadoras y sensores, la contrastación de hipótesis a través de la realización de dichas actividades experimentales, el análisis de datos y la formulación de regularidades. Todo el trabajo versara sobre la determinación parámetros fisicoquímicos de las sustancias que hacen parte de su cotidianidad y de su influencia en el entorno.

Tercera fase: evaluación

En esta etapa se llevará a cabo la aplicación de instrumentos a manera de postest que permitan determinar el alcance de la estrategia implementada en el desarrollo de competencias científicas espećficas como interpretar situaciones y establecer condiciones.

\section{Conclusiones}

Este trabajo pretende contribuir a la enseñanza de la química para formar estudiantes capaces de utilizar las competencias adquiridas en la educación, y poderse desenvolver en una sociedad de la información. 
Que comprendan y utilicen el conocimiento científico mediante la incorporación de herramientas de las NTIC que influyen y transforman el aprendizaje, al modificar de forma sustancial el contenido de un tema y la manera en que puede enseñarse y aprenderse, esto como una alternativa que favorezca, impulse y mejore la calidad de la educación química en el país.

Que nuestros estudiantes relacionan la noción de competencia y los conocimientos socialmente relevantes, con sus capacidades complejas, construidas desde la integración de saberes a partir del entomo y de otros desarrollados en diversos grados, lo que le permitirá y facilitara el desarrollo de las competencias científicas espećficas como la interpretación de situaciones, el establecer condiciones, y plantear hipótesis en la resolución de problemas.

Recordemos que la noción de competencia tiene relación con los fines educativos y significa que se trata de "desarrollar las habilidades y destrezas para saber qué hacer ante cambios de desafíos y problemas, deseables y no deseables que la sociedad le plantea a cada uno de los ciudadanos, estamos convencidas que el uso de la tecnología favorecerá este proceso motivando su participación en forma integral.

Y como esta sociedad del siglo XXI seguramente reafimará que aprender es la más importante fuente de riqueza y bienestar, de capacidad de competir y de cooperar. En consecuencia, cada institución educativa tiene que empezar por aceptar la necesidad de transformarse en una organización competitiva para facilitar el aprendizaje personal y colectivo ante el siglo XXI.

\section{Bibliografía}

Castiblanco A. (2001). Incorporación de Nuevas Tecnologías al Curnículo de matemáticas de la educación media de Colombia. II Fase: Profundización y Expansión.

García J. J. (2000). La Solución de Situaciones Problemáticas: una Estrategia Didáctica para la Enseñanza de la Química, en Revista Enseñanza de las Ciencias (18). 1.

Gil P. y Valdes C. (1996). La orientación de las prácticas de laboratorio como investigación: un ejemplo ilustrativo, en Revista Enseñanza de las Ciencias (14). 2

Hemández C. (2005). ¿Qué son las "Competencias Cient́ficas"? Universidad Nacional.

Sarmiento. Y. y Benavides S. (2007). Diseñar, experimentar, implementar y evaluar un software educativo en un ambiente de juego de roles relacionado con las pruebas de identificación de grupos funcionales de la química orgánica, para desarrollar competencias cognitivas. Universidad Pedagógica Nacional. 